\title{
Akt-mTOR signaling is involved in Notch-1-mediated glioma cell survival and proliferation
}

\author{
NENGJIANG ZHAO, YA GUO, MINGFANG ZHANG, LING LIN and ZHIHONG ZHENG \\ Department of Biochemistry and Molecular Biology, Research Center of Molecular Medicine, \\ Fujian Medical University, Fuzhou 350004, P.R. China
}

Received December 17, 2009; Accepted January 26, 2010

DOI: $10.3892 /$ or_00000782

\begin{abstract}
Both Notch signaling and Akt-mTOR signaling pathway are involved in glioma cell proliferation and survival. Previous studies have shown that Notch-1 is overexpressed in many glioma cell lines and primary human gliomas. Blocking of Notch signaling pathway can induces glioma cell apoptosis and growth suppression. However, the underlying molecular mechanism is not clear. We report that activation of the Notch pathway by intracellular domain of human Notch-1 (NIC-1) strongly activates Akt and promotes U251 glioma cell proliferation. Knockdown of Notch-1 by RNA interference suppresses Akt activation, reduces glioma cell growth rate and induce cell apoptosis. Following Notch-1 suppression, phosphorylated Akt and its downstream effector mTOR were reduced. Knockdown of Notch-1 also involves downregulation of anti-apoptotic protein MCL-1, in parallel with activation of apoptotic associate proteins PARP, caspase-9 and caspase- 3 . Our data demonstrate that Notch-1 can positively regulate Akt-mTOR pathways, which is associated with glioma cell proliferation and apoptosis. This also suggests a molecular mechanism for the inhibitory effect of Notch-1 RNA interference on glioma cell proliferation through Akt-mTOR signaling pathway.
\end{abstract}

\section{Introduction}

Glioma represents the most common primary brain tumors in adults. It is a major cause of morbidity and mortality in neurosurgical patients. Although great therapeutic efforts have been made, overall survival is still poor. Much research has focused on the understanding of the development of glioma, little is known about the cellular and molecular mechanisms that underlie glioma formation and progression. A number of signal transduction routes, such as the Notch signaling (1-3) and Akt-mTOR (4,5) pathways, seem to play

Correspondence to: Dr Zhihong Zheng, Department of Biochemistry and Molecular Biology, Research Center of Molecular Medicine, Fujian Medical University, Fuzhou 350004, P.R. China E-mail: zhzheng@mail.fjmu.edu.cn

Key words: Notch-1, glioma, Akt, mTOR an important role in the formation and growth of glioma. However, it is still unknown if Akt-mTOR activation has a role in the Notch-signaling pathway in glioma.

The Notch signaling pathway, a conserved cell-cell communication pathway, has been implicated in different developmental processes (6). Notch receptors on the surface of one cell bind to their ligands on the adjacent cell, leading to the proteolytic cleavage of the Notch intracellular domain (NICD). The NICD subsequently translocates to the nucleus, where it can associate with the DNA-binding protein $\mathrm{Su}(\mathrm{H})$ / RBP-JK/cbfl and activates transcription of Notch target genes (7). Cell communication and cell fate are controlled by Notch signaling (8). Notch-1 signaling dysfunction will results in a variety of developmental defects and adult pathologies $(6,9)$. Previous research demonstrated that Notch-1 is overexpressed in many glioma cell lines and primary human gliomas (1), block of Notch-1 could suppress proliferation in multiple glioma cell lines by arresting cell cycle and inducing apoptosis (3). However, the underlying molecular mechanisms remain to be fully understood.

The Akt-mTOR pathway has emerged as a crucial regulator of widely divergent cellular processes including proliferation, differentiation and apoptosis. Akt is a serine/threonine kinase, containing an amino terminal pleckstrin homology $(\mathrm{PH})$ domain, a central kinase domain and a carboxyl-terminal regulatory domain (10). Studies have shown that Akt can influence cell proliferation and cell cycle regulatory pathways by different signaling cascades. Downstream signaling of the PI3K/Akt pathway including p21/p27, nuclear factor-кB, caspase-9, caspase-3 and mTOR. mTOR plays a key role in downstream signaling of the Akt pathway through the regulation of cellular catabolism, proliferation, cell cycle, autophagy and apoptosis.

The precise mechanisms how Notch signaling regulates glioma survival remain poorly understood. Considering that Notch-1 signaling regulates EGFR and the blocking of Notch-1 induces glioma cell apoptosis (11), we hypothezised that Notch signaling affects glioma cell proliferation and survival by controlling the Akt/mTOR signaling pathway, because both pathways are widely known to govern cell proliferation and survival. In this study, we demonstrate that active Notch-1 increased p-Akt expression and blocking of Notch-1 suppressed Akt/mTOR activation. Our results suggest that Notch-1 signaling also regulate Akt pathway down targets p27 and cyclin D1. Accordingly, Akt/mTOR signaling was as a down target of Notch-1 signaling in glioma 
and Notch-1 block-induced apoptosis, at least in part, through suppressed Akt/mTOR signaling.

\section{Materials and methods}

Cell culture. Human glioblastoma cell line U251 was purchased from Cell Bank of Chinese Academic of Science (Shanghai, China). The cells were cultured at $37^{\circ} \mathrm{C}$ in a $5 \%$ humidified atmosphere in DMEM (Gibco) plus 10\% fetal calf serum.

Notch-1 RNA interference. The pENTRTM/U6 vector including U6 promoter and pLenti6/BLOCK-iT ${ }^{\mathrm{TM}}-\mathrm{DEST}$ were purchased from Invitrogen Inc. To construct the short hairpin RNA (shRNA) oligos, the Notch-1 (NM-017617) specific oligo sequences (5'-CGACGATTGTCCAGGAAA CAA-3') and scrambled control (5'-GTTCTCCGAACGTGT CACGT-3') were used. The oligonucleotides containing hairpin sequence were synthesized by Invitrogen Life Technologies (Guangzhou, China). The oligonucleotides were annealed and then cloned into pENTRTM/U6 plasmid to obtain an entry construction. The positive clones were verified by sequencing. A recombination reaction was performed between the pENTR/U6 entry construction and pLenti6/BLOCK-iT ${ }^{\mathrm{TM}}$ DEST vector and then the positive clones were confirmed by sequencing. The recombined plasmid and packaging mix were co-transfected with 293FT by Lipofectamine ${ }^{\mathrm{TM}} 2000$. Virus was harvested at $72 \mathrm{~h}$ after transfection and filtered through $0.45 \mu \mathrm{m}$ filters. Virus titers were calculated and concentrated virus were used to infect U251 glioma cells. Stably transfected cell clones were obtained from Blasticidin $(5 \mu \mathrm{g} / \mathrm{ml})$, selected and the effect of Notch-1 knockdown was confirmed by Western blot analysis.

NICD overexpression vector construction and cell transfection. The plasmid pNL-IRES2-EGFP is a generous gift of Dr Yanding Zhang and it originated from Dr Yiping Chen's Laboratory (Tulane University, USA). The cDNA encoding a constitutively active form of Notch-1 (NM-017617) intracellular domain (base pairs 5308-7665) was amplified by RT-PCR from U251 cells using forward primer: 5'-GCCACC ATGGTGCTGCTGTCCCGCAAG-3' and reverse primer: 5'-GCACACAGACGCCCGAAGG-3'. Notch-1 intracellular domain was cloned into pCR-XL-TOPO vector (Invitrogen, USA) and then digested with EcoRI and subcloned into

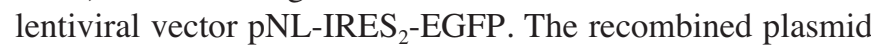
was confirmed by sequencing and co-transfected with $293 \mathrm{~T}$, VSVG and Help packaging Vectors. Lentiviruses were produced and concentrated to infect the U251 cells. The expression of Notch-1 intracellular domain was confirmed by Western blot analysis.

MTT assay. The growth rate of U251 cells was measured by MTT assay. Briefly, the Notch1-shRNA or control-shRNA lentivirus infected cells, expressing NICD or empty control lentivirus infected cells were plated into 96-well plates at $2 \times 10^{3}$ cells per well, respectively. At d1, d2, d3, d4, d5 and d6 after plating, $20 \mu \mathrm{l}$ MTT $(5 \mathrm{mg} / \mathrm{ml})$ was added to each well and the cells were incubated at $37^{\circ} \mathrm{C}$ for an additional 4 h. The reaction was then terminated by lysing the cells with
$200 \mu 1 \mathrm{DMSO}$ for $5 \mathrm{~min}$. The absorbance at $550 \mathrm{~nm}$ was measured using a Bio-Rad micro-plate reader. Each experiment was performed in triplicate and repeated at least three times.

Flow cytometric analysis of cell cycle kinetics and apoptosis. Cells were collected and fixed with ice-cold $70 \%$ ethanol, and stained with propidium iodide (PI) for $30 \mathrm{~min}$. Cell cycle was analyzed by EPICS-XL flow cytometer (BeckmanCoulter, USA).

Cell apoptosis was quantified by flow cytometeric analysis. In brief, cells were harvested and resuspended in the staining buffer and examined with Annexin V-FITC Apoptosis Detection kit (BioVision, USA) according to the manufacturer's instructions. The Annexin V-positive and PInegative cells were regarded as apoptotic cells.

Western blot analysis. Total proteins were extracted from infected U251 cells and subjected to $10 \%$ sodium dodecyl sulfate-polyacrylamide gel electrophoresis. Protein was transferred to PVDF membranes, which were blocked with TBST containing 5\% non-fat milk for $2 \mathrm{~h}$, and then incubated with anti-Notch-1-ICD (Chemicon, USA), anti-Akt, anti-p-Akt (Ser 473), anti-p-mTOR (Ser 2448), anti-p27 (Kip1) (Cell Signaling, USA); anti-caspase-9, anti-caspase-3, anti-MCL-1, anti-PARP-1 and GAPDH (Santa Cruz, USA) at $4^{\circ} \mathrm{C}$ overnight. After washing with TBST, the blots were incubated with HRP-labeled secondary antibodies for $2 \mathrm{~h}$ at room temperature. The blots were visualized by enhanced chemiluminescence (ECL) reagent kit (Beyotime, China) and quantified by densitometry.

Statistical analysis. All data are presented as mean \pm SEM of at least three independent experiments and analyzed using SPSS statistical package (Version 11.0). P-values were calculated using Student's t-test to compare results and $\mathrm{P}<0.05$ was considered as statistically significant.

\section{Results}

Knockdown of Notch-1 inhibited cell growth, induced cell cycle arrest and promoted apoptosis in U251 glioma cells. To test the effects of Notch-1 on maintenance of glioma cell proliferation and survival, we transfected U251 cells with Notch-1-shRNA or control shRNA expressing lentiviruses. Results showed that expression of Notch-1 protein in its RNA interference cells was much lower as confirmed by Western blot analysis (Fig. 1A).

The proliferation activity of U251 cells were examined by MTT assay. After knockdown of Notch-1 expression by RNA interference, as shown in Fig. 1C, the growth rate of cells was inhibited significantly compared to control.

The results of flow cytometry showed that knockdown of Notch-1 in U251 cells resulted in the decrease of the S phase fraction and arrest of cells in the G0/G1 phase (Fig. 2A), whereas the number of apoptotic cells evaluated by Annexin V labeling was significantly increased (Fig. 1E).

Overexpression of active Notch-1 promotes cell proliferation and increase cells in $S$ phase. To further confirm the role of 

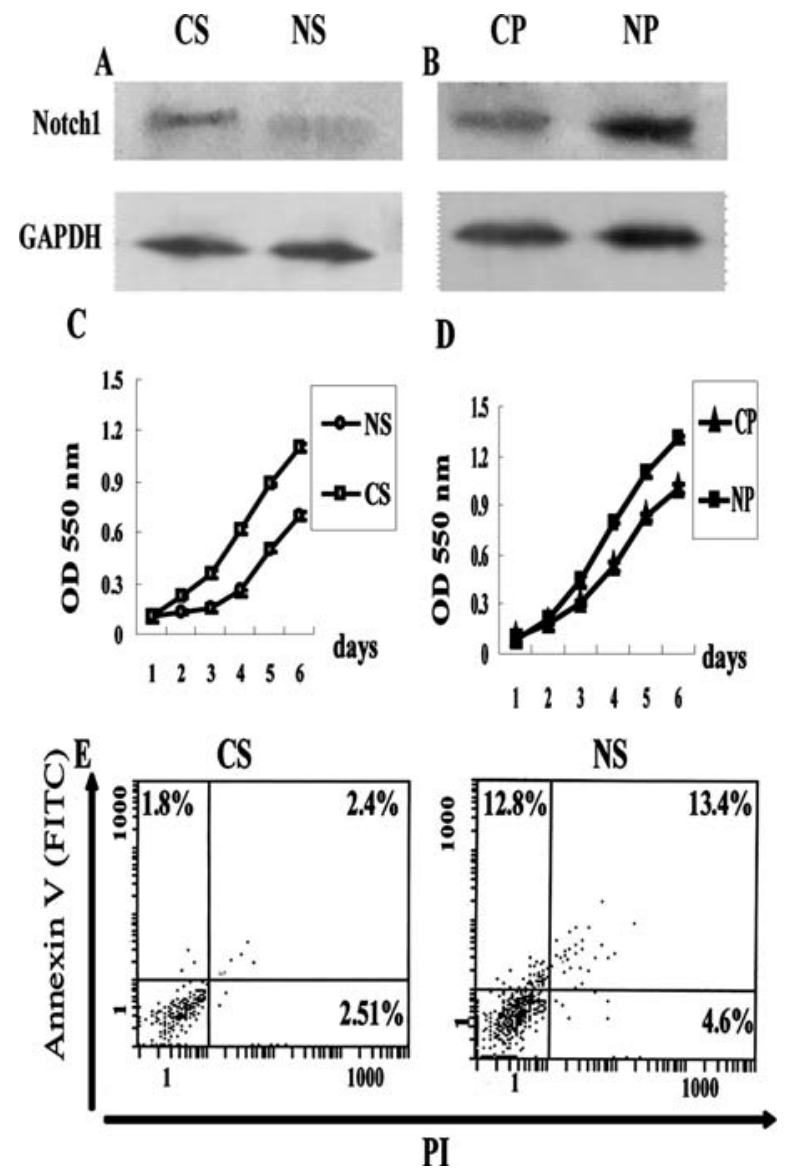

Figure 1. Effects of Notch-1 on glioma cell proliferation and apoptosis (CS, infected with control shRNA lentivirus; NS, infected with Notch-1 shRNA lentivirus; $\mathrm{CP}$, infected with control lentivirus; NP, infected with expressing NICD lentivirus). The expressions of NICD in U251 cells were analyzed by Western blotting. (A) Notch-1 was down-regulated by Notch-1 RNA interference in U251 cells compared with control cells. (B) NICD as active Notch-1 was overexpression in U251 cells infected with NICD lentivirus. Effects on cells growth were examined by MTT assay. (C) Cell growth was inhibited by Notch-1 knockdown. (D) Overexpression of NICD stimulated cell growth. Cells apoptosis were examined by flow cytometry with Annexin V and PI double staining. (E) Notch1 knockdown cell apoptosis ratio was increased compared with control.

Notch-1 in U251 cell proliferation and survive, the U251 cells were transfected with human NICD-expressing lentivirus or empty control lentivirus alone. We used Western blot analysis to confirm the overexpression of Notch-1-ICD in transfected cells and found they had higher level expression of Notch-1-ICD (Fig. 1B). Overexpression of active Notch-1 led to the promotion of cell proliferation when compared to control (Fig. 1D). Overexpression of active Notch-1 also led to the promotion of cell cycles. The percentage of cells in $\mathrm{S}$ phase was increased from $23.6 \%$ in the control to $30.5 \%$ in overexpressing NICD cells (Fig. 2A).

Effects of Notch-1 on cell cycle associated protein expression. We have demonstrated that knockdown of Notch-1 inhibited cell transition from G1 phase to S phase and active Notch-1 promoted the U251 cell S phase entry. To further elucidate how Notch-1 affected cell cycle distribution, p27 and cyclin D1 which are important in controlling $\mathrm{G} 1$ to $\mathrm{S}$ phase were detected by Western blot analysis. As shown in Fig. 2B, the

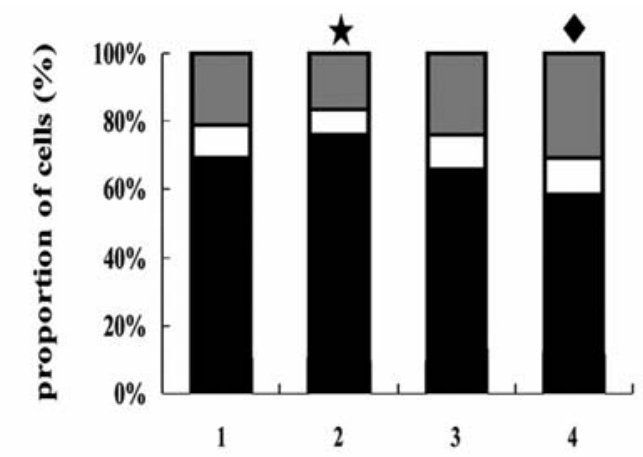

\begin{tabular}{|c|c|c|c|c|}
\hline$S$ & $22.3 \pm 0.6$ & $16.3 \pm 0.9$ & $23.5 \pm 0.3$ & $27.8 \pm 2.5$ \\
\hline G2/M & $7.9 \pm 1.3$ & $6.4 \pm 0.4$ & $8.7 \pm 0.3$ & $10.0 \pm 0.7$ \\
\hline$\overline{G 0 / G 1}$ & $69.6 \pm 0.9$ & $77.2 \pm 1.0$ & $67.7 \pm 3.6$ & $62.1 \pm 3.2$ \\
\hline
\end{tabular}
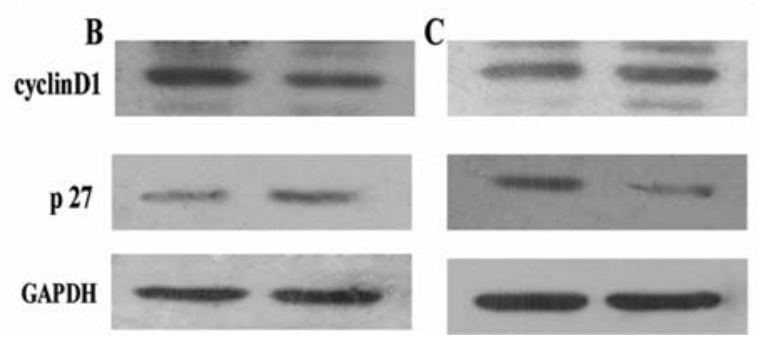

Figure 2. The effect of Notch-1 on cell cycle and cell cycle associated protein expression. (A) Cell cycle was analysed by flow cytometry. S phase cells were decreased following Notch-1 knockdown ( ${ }^{\star} \mathrm{P}<0.05$ vs. CS $)$. Overexpression of NICD promoted $\mathrm{S}$ phase entry ( ${ }^{\star} \mathrm{P}<0.05$ vs. CP). (B) The protein expressions were analysed by Western blotting. Notch-1 knockdown decreased cyclin D1 and increased p27 expression. (C) Overexpression of NICD increased cyclin D1 and decreased p27 expression.

protein levels of cyclin D1 decreased and the cell cycle inhibitor p27 increased following Notch-1 knockdown. Notch-1 activation increased cyclin D1 expression and decreased p27 expression (Fig. 2C).

Effects of Notch-1 on cell apoptosis-associated protein expression. We have demonstrated that knockdown of Notch-1 induced U251 cell apoptosis. To further elucidate how Notch-1 regulation affect apoptosis, MCL-1, PARP-1, caspase- 9 and caspase-3, which are associated with apoptosis, were detected by Western blot analysis. As shown in Fig. 3, the expression levels of PARP-1, caspase-9 and caspase-3 protein were decreased following Notch-1 knockdown and increased after Notch-1 activation. The expression of antiapoptotic protein MCL-1 was decreased following Notch-1 knockdown.

Notch-1 regulation of Akt and downstream effector mTOR in U251 glioma cells. Akt has emerged as a crucial regulator of cellular processes including proliferation, differentiation and apoptosis. Due to Notch-1 affected cell cycle, we predict that Akt pathway may participate in this process. Akt target proteins were detected after Notch-1 knockdown and overexpression. The results showed that the levels of phosphorAkt, and phosphor-mTOR were down-regulated following Notch-1 knockdown. However, the level of total Akt protein 


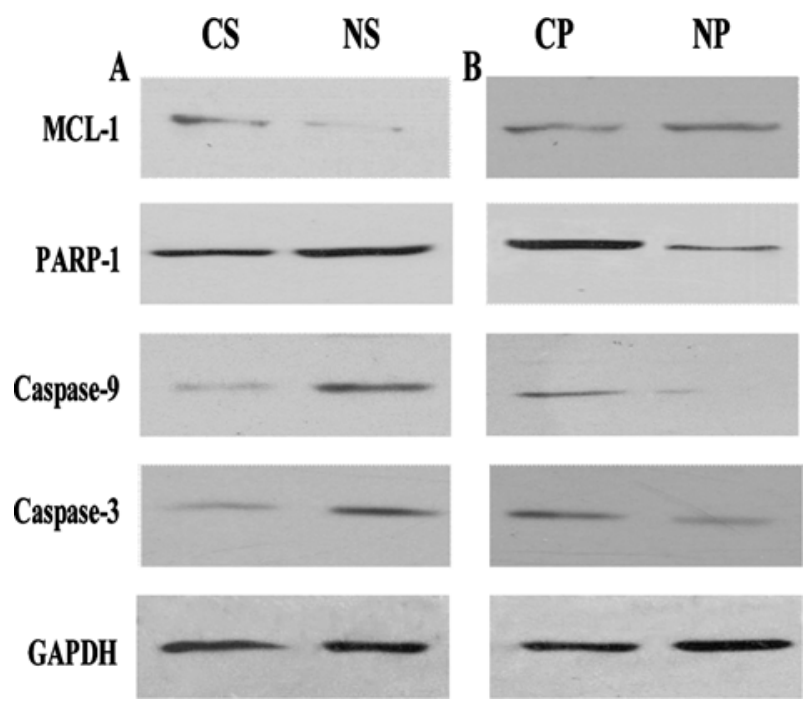

Figure 3. Western blot analysis of apoptosis-associated protein expression (A) In Notch-1 RNA interference cells, expression of MCL-1 was decreased, PARP-1, caspase-9 and caspase-3 were increased; (B) In Notch-1 activated cells, expression of MCL-1 was increased; PARP-1, caspase-9 and caspase- 3 were decreased.

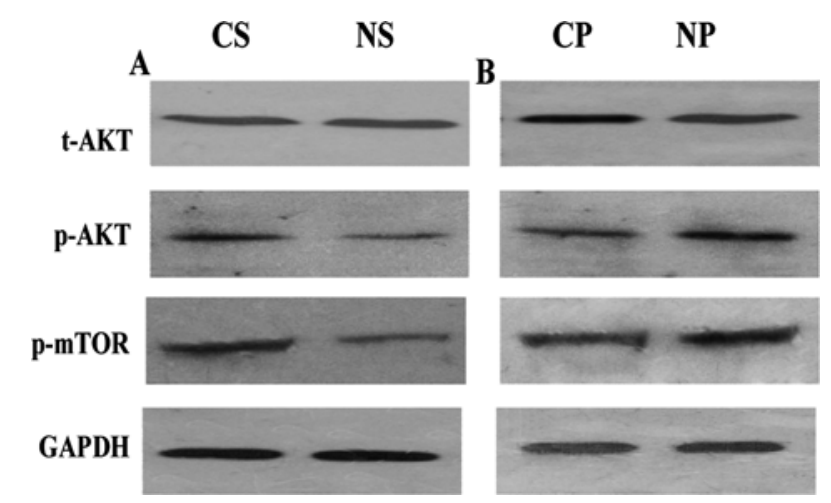

Figure 4. Western blot analysis of Akt and its downstream protein expression. (A) In Notch-1 RNA interference cells, p-Akt and p-mTOR were decreased; (B) In Notch-1 activated cells, p-Akt and p-mTOR were increased.

was not affected (Fig. 4). Thus, these results indicated that silencing of Notch-1 inhibited Akt activation but not Akt protein expression.

\section{Discussion}

Despite aggressive treatment strategies, patients with glioma still have a dismal prognosis, which highlights the need to understand the molecular mechanism responsible for glioma formation and progression. Various signaling cascades are involved in the process, including Notch and Akt-mTOR signaling. It was found that there is cross-talk between Notch and Akt signaling $(12,13)$, but the relation in glioma cells is not clear. To further explore the interactions between Notch and Akt signaling in glioma cells proliferation and survival, we investigated the overexpression and low-expression of Notch-1 in U251 glioma cells and found Notch-1 could regulate Akt activation which associated with glioma proliferation and apoptosis.
The Notch signaling pathway is an evolutionarily conserved signaling system which has been shown to play important roles in many different biological processes including proliferation, differentiation, survival and apoptosis. Previous studies have provided that aberration of the Notch-signaling pathway contributes to the formation, progression and prognosis of glioma $(2,14)$. Overexpression of Notch-1 and its ligands Delta-like-1 and Jagged-1 are present in many glioma cell lines and primary human gliomas and targeting Notch signaling may provide novel therapeutic targets (15). We found that Notch-1 activation could promote U251 cell proliferation and survival and increase the percentage of cells in S phase. Furthermore, knockdown of Notch-1 induced cell cycle arrest and apoptosis through down-regulated expression of cell cycle-associated proteins cyclin D1 and anti-apoptotic proteins MCL-1, increased the expression of apoptotic protein caspase-9, -3 and PARP-1. PARP-1 is a DNAbinding enzyme involved in modulation of DNA-repairmediated resistance to cytotoxic therapy. PARP-1 inhibition is associated with increased sensitivity to DNA-alkylating agents, topoisomerase I poisons and ionising radiation (16). Notch-1 may be involved in radio-chemotherapy resistance through regulation of PARP-1. It was found that the Notch ligand Delta-1 can inhibit apoptosis by reducing the cleavage of PARP-1 (17). Selective ablation of Notch3 in hepatocellular carcinoma enhances the doxorubicin death promoting effect (18). The inhibition of phosphatidylinositol-3-OH kinase/Akt signaling impairs DNA repair in glioblastoma cells following ionizing radiation (19). In this study, we first suggested that block Notch-1 in glioma cells can repress Akt and PARP-1. Our research also indicates that Notch-1 acts as an oncogene in glioma and is probably associated with radiochemotherapy resistance.

The Akt is known as an important downstream mediator of the PI3K signaling pathway in glioma. Akt is activated by phosphorylation. Phosphor-Akt regulates the function of many cells involved in metabolism, proliferation and apoptosis (20). Recent evidence indicated that Akt as an oncogene is frequently active in many types of human cancers $(10,21)$. At the same time, studies defined the importance of the Akt pathway in G1/S cell cycle progression. Accumulating evidence suggests that Akt can regulate cyclin D1 (22), its overexpression results in shortened cell cycle progression and thereby contributes directly to tumor progression. Akt also regulated p27(Kip1), a major cyclin/CDK inhibitor, activation. Akt can phosphorylate p27 and results in p27 degradation or cytoplasmic location, so that cyclin/CDK are activated and promote cell cycle progression $(23,24)$. Akt pathway also has been shown to regulate apoptosis through procaspases. Caspase- 9 acts as an initiator and an effector of apoptosis. Akt can phosphorylate procaspase- 9 and inhibit apoptosis induction (25). Akt has been shown to affect cell cycle progression and proliferation, also to possess a potential function in tumorigenesis and chemoresistance (26). Our results showed that silencing of Notch-1 caused a decrease of phospho-Akt but not total Akt. Reduced phosphor-Akt led to a decrease of phosphor-mTOR, cyclin D1, caspase-9 and caspase-3, and up-regulation of p27. These results suggest that the mechanism of Notch-1 gene blockinduced growth inhibition was achieved with inhibition of 
Akt signaling and it down-modulates Akt-dependent survival pathways in U251 glioma cells. However, how silencing of Notch-1 inhibits Akt pathway remains to be elucidated.

The canonical EGFR signaling network has attracted extensive studies recently due to its involvement in cancer growth, tumor progression, and its potential as a target for development of pharmaceutical inhibitors during cancer treatment. Amplification of the EGFR gene represents the important molecular genetic alteration identified in human glioma. Previous research found that blocking Notch-1 suppressed the transcription of the epidermal growth factor receptor (11). It should be noted that the transcriptional upregulation of EGFR by Notch activity is probably less potent than that from EGFR amplification. EGFR amplification may increase its expression by 10 - to 40 -fold, potentially overwhelming any effects from Notch regulation of EGFR, so blocking EGFR is not enough to block glioma growth. But we found that Akt, the major component of EGFR downstream was depressed by Notch-1 RNA interference. Notch-1 knockdown also depressed mTOR and correlated downstream components. Akt is an important target of EGFR and as a 'molecular switch' of cell proliferation and growth, but Akt activation is both dependent and not dependet on EGFR. In this study, our results indicate that mTOR and Akt were identified as downstream targets of Notch-1 and that Notch-1 could be used in a treatment aimed at blocking both Akt and mTOR driven glioma cell proliferation.

Accordingly, silencing of Notch-1 slows cell growth and enhances apoptosis through inhibition of Akt signaling pathway in glioma. We report for the first time that mTOR signaling pathway was involved in the effects induced by Notch-1 RNA interference in U251 cells. Our findings confirm Notch-1 as a rational therapeutic target in glioma.

\section{Acknowledgements}

We thank Dr Yanding Zhang (Fujian Normal University) for providing plasmid pNL-IRES2-EGFP. This study was supported by the Natural Science Foundation (C0810017) of Fujian Province in China and Research Fund (JS06008) from Fujian Medical University.

\section{References}

1. Purow BW, Haque RM, Noel MW, Su Q, Burdick MJ, Lee J, Sundaresan T, Pastorino S, Park JK, Mikolaenko I, Maric D, Eberhart CG and Fine HA: Expression of Notch-1 and its ligands, Delta-like-1 and Jagged-1, is critical for glioma cell survival and proliferation. Cancer Res 65: 2353-2363, 2005.

2. Zhang XP, Zheng G, Zou L, Liu HL, Hou LH, Zhou P, Yin DD, Zheng QJ, Liang L, Zhang SZ, Feng L, Yao LB, Yang AG, Han $\mathrm{H}$ and Chen JY: Notch activation promotes cell proliferation and the formation of neural stem cell-like colonies in human glioma cells. Mol Cell Biochem 307: 101-108, 2008.

3. Xu P, Qiu M, Zhang Z, Kang C, Jiang R, Jia Z, Wang G, Jiang H and $\mathrm{Pu} \mathrm{P}$ : The oncogenic roles of Notch1 in astrocytic gliomas in vitro and in vivo. $\mathrm{J}$ Neurooncol 97: 41-51, 2010.

4. Annovazzi L, Mellai M, Caldera V, Valente G, Tessitore L and Schiffer D: mTOR, S6 and AKT expression in relation to proliferation and apoptosis/autophagy in glioma. Anticancer Res 29: 3087-3094, 2009.

5. McBride SM, Perez DA, Polley MY, Vandenberg SR, Smith JS, Zheng S, Lamborn KR, Wiencke JK, Chang SM, Prados MD, Berger MS, Stokoe D and Haas-Kogan DA: Activation of PI3K/ mTOR pathway occurs in most adult low-grade gliomas and predicts patient survival. J Neurooncol 97: 33-40, 2010.
6. Lasky JL and $\mathrm{Wu} \mathrm{H}$ : Notch signaling, brain development, and human disease. Pediatr Res 57: R104-R109, 2005.

7. Hansson EM, Lendahl U and Chapman G: Notch signaling in development and disease. Semin Cancer Biol 14: 320-328, 2004.

8. Lai EC: Notch signaling: control of cell communication and cell fate. Development 131: 965-973, 2004

9. Bolos V, Grego-Bessa J and De la Pompa JL: Notch signaling in development and cancer. Endocr Rev 28: 339-363, 2007.

10. Morgensztern D and McLeod HL: PI3K/Akt/mTOR pathway as a target for cancer therapy. Anticancer Drugs 16: 797-803, 2005.

11. Purow BW, Sundaresan TK, Burdick MJ, Kefas BA, Comeau LD, Hawkinson MP, Su Q, Kotliarov Y, Lee J, Zhang W and Fine HA: Notch-1 regulates transcription of the epidermal growth factor receptor through p53. Carcinogenesis 29: 918-925, 2008.

12. Liu ZJ, Xiao M, Balint K, Smalley KS, Brafford P, Qiu R, Pinnix CC, Li X and Herlyn M: Notch1 signaling promotes primary melanoma progression by activating mitogen-activated protein kinase/phosphatidylinositol 3-kinase-Akt pathways and up-regulating N-cadherin expression. Cancer Res 66: 4182-4190, 2006.

13. Gutierrez A and Look AT: NOTCH and PI3K-AKT pathways intertwined. Cancer Cell 12: 411-413, 2007.

14. Grandbarbe L, Bouissac J, Rand M, De Angelis MH, ArtavanisTsakonas S and Mohier E: Delta-Notch signaling controls the generation of neurons/glia from neural stem cells in a stepwise process. Development 130: 1391-1402, 2003.

15. Kanamori M, Kawaguchi T, Nigro JM, Feuerstein BG, Berger MS, Miele L and Pieper RO: Contribution of Notch signaling activation to human glioblastoma multiforme. J Neurosurg 106: 417-427, 2007.

16. Peralta-Leal A, Rodriguez-Vargas JM, Aguilar-Quesada R, Rodriguez MI, Linares JL, De Almodovar MR and Oliver FJ: PARP inhibitors: new partners in the therapy of cancer and inflammatory diseases. Free Radic Biol Med 47: 13-26, 2009.

17. Murata-Ohsawa M, Tohda S, Sakano S and Nara N: The Notch ligand, Delta-1, partially inhibits GM-CSF-induced differentiation and apoptosis along with reducing the cleavage of PARP in U937 cells. Int J Mol Med 13: 419-423, 2004

18. Giovannini C, Gramantieri L, Chieco P, Minguzzi M, Lago F, Pianetti S, Ramazzotti E, Marcu KB and Bolondi L: Selective ablation of Notch3 in HCC enhances doxorubicin's death promoting effect by a p53-dependent mechanism. J Hepatol 50: 969-979, 2009.

19. Kao GD, Jiang Z, Fernandes AM, Gupta AK and Maity A: Inhibition of phosphatidylinositol-3-OH kinase/Akt signaling impairs DNA repair in glioblastoma cells following ionizing radiation. J Biol Chem 282: 21206-21212, 2007.

20. Nicholson KM and Anderson NG: The protein kinase B/Akt signalling pathway in human malignancy. Cell Signal 14: 381-395, 2002 .

21. Hambardzumyan D, Becher OJ, Rosenblum MK, Pandolfi PP, Manova-Todorova $\mathrm{K}$ and Holland EC: PI3K pathway regulates survival of cancer stem cells residing in the perivascular niche following radiation in medulloblastoma in vivo. Genes Dev 22 . 436-448, 2008.

22. Takuwa N, Fukui Y and Takuwa Y: Cyclin D1 expression mediated by phosphatidylinositol 3-kinase through mTORp70(S6K)-independent signaling in growth factor-stimulated NIH 3 T3 fibroblasts. Mol Cell Biol 19: 1346-1358, 1999.

23. Shin I, Yakes FM, Rojo F, Shin NY, Bakin AV, Baselga J and Arteaga CL: PKB/Akt mediates cell-cycle progression by phosphorylation of p27(Kip1) at threonine 157 and modulation of its cellular localization. Nat Med 8: 1145-1152, 2002.

24. Fiano V, Ghimenti C, Imarisio S, Silengo L and Schiffer D: PAkt, cyclin D1 and p27/Kip.1 in glioblastomas with and without EGFR amplification and PTEN mutation. Anticancer Res 24: 2643-2647, 2004.

25. Allan LA and Clarke PR: Apoptosis and autophagy: regulation of caspase-9 by phosphorylation. FEBS J 276: 6063-6073, 2009.

26. Abedini MR, Muller EJ, Bergeron R, Gray DA and Tsang BK: Akt promotes chemoresistance in human ovarian cancer cells by modulating cisplatin-induced, p53-dependent ubiquitination of FLICE-like inhibitory protein. Oncogene 29: 11-25, 2010. 